\title{
EFECTO DE BIOFERTILIZANTE Y ESTRÉS POR HIDROMORFÍA EN VITROPLANTAS DE CAÑA DE AZÚCAR, VARIEDAD C120-78
}

\author{
EFFECT OF A BIOFERTILIZER AND THE RESPONSE TO THE \\ WATERLOGGING STRESS IN SUGARCANE VITROPLANTS, \\ VARIETY C120-78
}

\author{
Sergio Rodríguez y Jorge Romero ${ }^{2}$ \\ Recibido para evaluación: Abril 4 de 2007 - Aceptado para publicación: Octubre 25 de 2007
}

\section{RESUMEN}

El sobrehumedecimiento del suelo agravado por los efectos de cambio climático global es un fenómeno que afecta a plantas y microorganismos en varias regiones del mundo. La presencia de actividad endófita en la caña de azúcar de cepas de Azospirillum brasilense puede constituir una alternativa sustentable debido a la distribución universal de esta bacteria y a sus variados efectos benéficos en el crecimiento y desarrollo de las plantas. En un suelo Typic Haplustert del Valle del Río Cauto, en la región oriental de Cuba se plantó un experimento en bloques al azar, con la variedad de caña de azúcar C120-78, obtenidas por cultivo "in vitro", inoculadas y sin inocular con la bacteria Azospirillum brasilense, cepa 8 del INICA, en condiciones normales y con el suelo sobresaturado de agua, con el objetivo de determinar la influencia que ejerce esta cepa bacteriana en indicadores del crecimiento y desarrollo de esta variedad, en ciclo de caña planta. Se demostró que para el pol en caña, t caña ha ${ }^{-1}$, t pol ha ${ }^{-1}$ y desarrollo del sistema radical, a los doce meses de edad, la presencia de la bacteria en el suelo no sobresaturado de agua mostró los mejores resultados, lo que demuestra que la carencia de oxígeno en el suelo es un elemento limitante para la actividad de esta cepa bacteriana y el normal crecimiento y desarrollo de la caña de azúcar.

Palabras clave: Azospirillum, sobrehumedecimiento, rendimiento, sistema radical.

\begin{abstract}
The soil flooding increased by the effects of global climatic change is a phenomenon that affected plants and microorganisms in many regions of the world. The presence of endophyte activity in sugarcane of Azospirillum brasilense strain's can constitute a sustainable alternative due to the universal distribution of this bacterium and to its various beneficial effects on plant growth. In a Typic Haplustert soil of the Cauto's River Valley, in the east region of Cuba, an experiment was established using a randomized blocks design, variety C120-78, obtained by in vitro culture, inoculated and without inoculating with Azospirillum brasilense

\footnotetext{
${ }^{1}$ Universidad de Granma, Facultad de Ciencias Agrícolas, Centro de Estudios Biotecnología Vegetal, Cuba, Email: srrguez@udg.co.cu

${ }^{2}$ Corporación Colombiana de Investigaciones Agropecuarias (CORPOICA), Colombia, Apartado Aéreo 602, Montería, Email: jromero@corpoica.org.co
} 
strain 8 from INICA, under normal conditions and with flooded soil, with the objective of determining the influence of this bacterial strain on growth of the sugar cane plant cycle. It was demonstrated that the pol percentage cane, cane ton ha ${ }^{-1}$, pol ton $\mathrm{ha}^{-1}$ and the radical system growth at the twelve months of age, the presence of the bacteria in non flooded soil showed the best results, demonstrated that the low level oxygen in the soil is a restrictive element for the activity of this bacterial strain and the normal growth of the sugarcane.

Key words: Azospirillum, flooding, yield, radical system.

\section{INTRODUCCIÓN}

Las Poaceas, donde está incluida la caña de azúcar (Saccharum spp. Híb.), se encuentran ampliamente distribuidas en diferentes regiones geográficas debido a su versatilidad edafoclimática. En muchas de estas áreas existen condiciones que favorecen la sobresaturación de agua en el suelo, alterando el equilibrio agua - aire necesario para plantas y microorganismos, con afectaciones en la respuesta agroindustrial de la caña de azúcar (Deren et al., 1993).

La práctica de aplicar inoculantes microbianos en la agricultura, constituye una alternativa promisoria que ha alcanzado auge internacional (Pérez y Casas, 2005). Uno de los más empleados ha sido el género Azospirillum, con capacidad de fijar nitrógeno atmosférico en condiciones microaeróbicas y producir sustancias estimuladoras del crecimiento (Eckert et al., 2001).

De las aplicaciones del cultivo in vitro, la multiplicación de plantas es la más usada, su premisa consiste en que las plantas resultantes del proceso sean fenotípica y genotípicamente idénticas a la planta donante (INICA, 2002). La micropropagación de la caña de azúcar por un lado resuelve en parte los problemas sanitarios que se presentan durante la producción de semilla utilizando los métodos tradicionales y por otra parte incrementa los coeficientes de multiplicación, que impide una rápida propagación de plantas libres de enfermedades (INICA, 2002). Esta investigación tiene como objetivo, determinar la influencia que ejerce la bacteria Azospirillum brasilense, cepa 8 del INICA, sobre indicadores del crecimiento y desarrollo de la caña de azúcar, variedad C12078 obtenida por cultivo in vitro, en condiciones normales y con el suelo sobresaturado de agua.

\section{MATERIALES Y MÉTODOS}

En un suelo Typic Haplustert (Driessen et al., 2001; Soil Survey Staff, 2003) ubicado en el antiguo bloque experimental de la Empresa Azucarera "Grito de Yara" de la provincia de Granma, Cuba, se desarrolló el experimento de campo. El material vegetal empleado fueron vitroplantas de caña de azúcar de la variedad C120-78, con sus partes aéreas y radicales suficientemente desarrolladas, sumergidas en una suspensión con la presencia de la bacteria Azospirillum brasilense, cepa 8

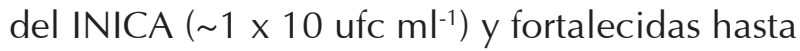
los 45 días antes de ser llevadas a campo, en cajas de polieturano de 120 orificios, con la presencia de un sustrato compuesto por una mezcla de suelo y cachaza (50:50).

Los tratamientos bajo estrés por sobrehumedecimiento se manejaron después de los noventa días de plantadas las vitroplantas, una vez terminado el ahijamiento. Cada diez días se le aplicó una lámina de agua por gravedad que mantuviera la humedad del suelo por un período no mayor de tres días, estas parcelas estaban protegidas por un dique para mantener esta condición. La conducción del agua se efectuó por 
tuberías de un sistema San Cristóbal, aplicándola por gravedad a cada parcela. Este estrés se suspendió tres meses antes de la cosecha, para facilitar esta labor. Para ambas condiciones durante los primeros noventa días se aplico riego por gravedad para garantizar la adaptación y supervivencia de las vitroplantas a las condiciones de campo cada vez que estas lo requerían, con una norma neta parcial de riego de $237 \mathrm{~m}^{3} \mathrm{ha}^{-1}$, cada 12 días. Fueron plantadas en el mes de enero y la evaluación y cosecha se realizó a los 12 meses como ciclo de caña planta.

El diseño empleado en campo fue bloques al zar con cuatro réplicas de $48 \mathrm{~m}^{2}$ cada una, resultando los siguientes cuatro tratamientos que se denotan a continuación:

Tratamiento I: Suelo sin exceso de agua y vitroplantas sin Azospirrillum.

Tratamiento II: Suelo sin exceso de agua y vitroplantas con Azospirrillum.

Tratamiento III: Suelo bajo estrés por sobrehumedecimiento sin Azospirrillum.

Tratamiento IV: Suelo bajo estrés por sobrehumedecimiento con Azospirrillum.

\section{Porcentaje de pol en caña y toneladas de pol por hectárea}

La primera se determinó por estimación en el Polarímetro, toneladas de caña por hectárea por pesaje directo en campo y la segunda por estimación de acuerdo a la metodología del INICA (2002).

\section{Profundidad radical}

El muestreo y estudio del sistema radical se realizó unos días antes de la cosecha, tomando en consideración la técnica del monolito, descrita, por Kolensnikov (1971), considerando las profundidades de muestreo recomendadas para caña de azúcar por Krautman (1959). Se graficó la profundidad alcanzada por el setenta por ciento de la masa fresca del sistema radical, considerada la zona más activa (Humbert, 1965).
Las variables evaluadas cumplieron en todos los casos las premisas del análisis de varianza, el ajuste a la distribución normal (Kolmogorov - Smirnov) y la prueba de Bartlett para la homogeneidad de las varianzas. Se empleó análisis de varianza de clasificación doble por efectos fijos, con Tukey como prueba de comparación múltiple de medias y una probabilidad del 0.05. Se muestran las medias y sus errores estándar en las mismas unidades de medida que las variables originales, así como el coeficiente de variación, en porcentaje. Se empleó además el Análisis de Componentes Principales (Hotelling, 1933) como técnica de reducción de dimensiones asumiendo como individuos los cuatro tratamientos y los cuatro indicadores evaluados como variables. El análisis se representó en un gráfico del tipo biplot Gabriel (1971). Se determinaron los autovalores, para conocer la variabilidad explicada, a partir de una matriz de correlaciones para estandarizar los datos, al estar las variables en unidades de medida diferentes, de acuerdo a la recomendación de Morrison (1979). Fueron incluidos dentro del análisis los Árboles de Recorrido Mínimo (Gower y Ross, 1969), para mostrar la menor distancia o similitud entre dos tratamientos en el espacio multivariado. Para el procesamiento estadístico de los datos se empleó el Paquete Estadístico SAS (2000).

\section{RESULTADOS Y DISCUSIÓN}

Con relación al porcentaje de pol en caña (Tabla 1) el análisis arrojó diferencias significativas entre los cuatro tratamientos. El tratamiento dos que consistió de el suelo sin exceso de agua y la presencia de la bacteria Azospirillum brasilense, alcanzó los mayores valores, significativamente superiores al tratamiento I, en el cual el suelo se encontraba sin exceso de agua y sin la presencia de Azospirillum brasilense. Los menores porcentajes de pol en caña correspondieron a los tratamientos III y IV 
Tabla 1: Respuesta de la variedad C120-78 en las variables pol en caña, t caña ha-1 y t pol ha-1, con relación a los tratamientos a los 12 meses de edad en ciclo de caña planta.

\begin{tabular}{|c|c|c|c|c|c|c|}
\hline \multirow{2}{*}{ Tratamientos } & \multicolumn{2}{|c|}{ Pol en caña (\%) } & \multicolumn{2}{|c|}{ t caña $h a^{-1}$} & \multicolumn{2}{|c|}{ t pol ha ${ }^{-1}$} \\
\hline & Media & Significancia & Media & Significancia & Media & Significancia \\
\hline 1 & 16.83 & $\mathrm{~b}$ & 46.10 & $\mathrm{~b}$ & 7.76 & $\mathrm{~b}$ \\
\hline II & 17.55 & $\mathrm{a}$ & 51.57 & $\mathrm{a}$ & 9.05 & $\mathrm{a}$ \\
\hline III & 14.47 & $\mathrm{C}$ & 41.53 & C & 6.01 & C \\
\hline IV & 14.83 & C & 42.07 & $\mathrm{C}$ & 6.24 & C \\
\hline Media $\pm \mathrm{EE}$ & \multicolumn{2}{|c|}{$15.92 \pm 0.3306$} & \multicolumn{2}{|c|}{$45.32 \pm 1.7103$} & \multicolumn{2}{|c|}{$7.27 \pm 0.4115$} \\
\hline CV $(\%)$ & \multicolumn{2}{|c|}{7.15} & \multicolumn{2}{|c|}{8.35} & \multicolumn{2}{|c|}{10.03} \\
\hline
\end{tabular}

Letras diferentes indican diferencias significativas al 0,05 empleando Tukey.

donde predominó la sobresaturación de agua en el suelo, aún con la presencia de la bacteria.

Los menores porcentajes de pol en caña encontrados en condiciones de sobresaturación de agua, son atribuidos al efecto que induce este tipo de estrés en la calidad de los jugos de los tallos de la caña de azúcar (Fida et al., 2003), debido a que este indicador es regulado por los genotipos y el ambiente imperante, estando la humedad del suelo entre los más influyentes (Inman-Bamber et al., 2004), debido a que el proceso de maduración de este cultivo no siempre va paralelo con la edad, por otro lado, de mantenerse el agua abundante en el suelo, la planta nunca madura completamente, debido a que el estímulo de la maduración se debe a un déficit de humedad acumulativo en el suelo (Martín et al., 1987). Además, la carencia de oxígeno en el suelo reduce la producción de ATP en la célula y la glucólisis se convierte en la única ruta para la producción de ATP (Drew, 1997), la cual es menos eficiente, lo que requiere del consumo de cantidades de carbohidratos elevadas por la planta.

Para el rendimiento agrícola (t caña $h a^{-1}$ ) y agroindustrial (t pol $\mathrm{ha}^{-1}$ ), los tratamientos alcanzaron resultados semejantes con relación al porcentaje de pol en caña en cuanto al orden de mérito de los mismos, basado en la dependencia que tiene el rendimiento agroindustrial de las otras dos variables. Las variaciones que mostró el rendimiento agrícola pueden haber estado asociadas a las diferentes profundidades que alcanzó el sistema radical (Figura 1), debido a que coincidentemente el tratamiento II, que alcanzó el mayor rendimiento agrícola fue el que además la masa fresca del $70 \%$ de su sistema radical penetró a mayor profundidad. Se resalta el efecto inhibidor sobre el desarrollo en profundidad del sistema radical provocado por la sobresaturación de agua en el suelo, reflejado en los tratamientos III y IV, Io

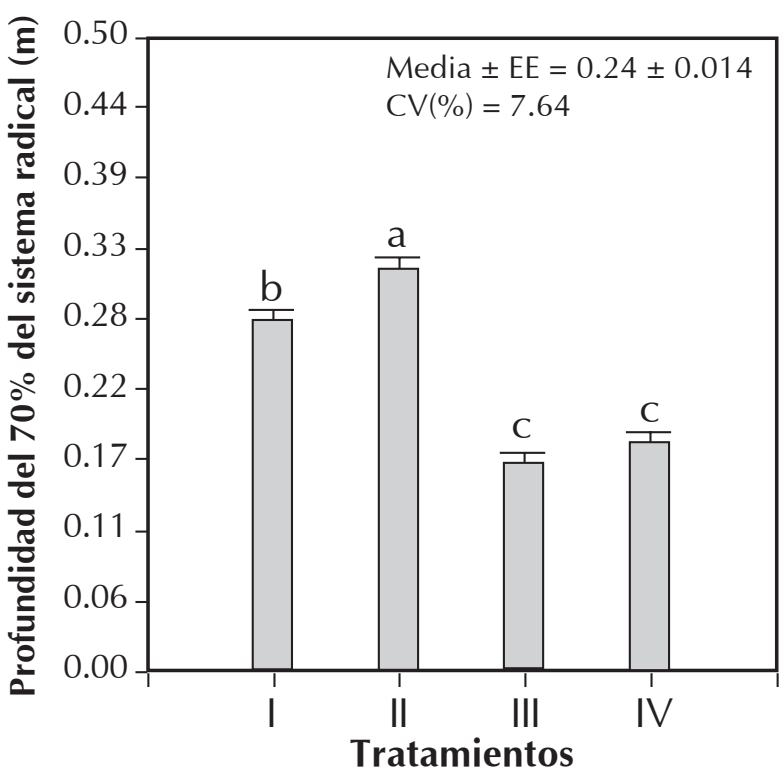

Figura 1. Profundidad que alcanzó el $70 \%$ de la masa fresca del sistema radical en los cuatro tratamientos en el ciclo de caña planta. 
cual es una limitante para la absorción de agua y nutrientes por la planta. Esta reducción del sistema radical en profundidad en suelos sobrehumedecidos y que limita los rendimientos agrícolas de la caña de azúcar, se relaciona también con la acumulación de sustancias tóxicas en las raíces que inhiben la absorción de nutrientes y producen descensos en sus disponibilidades en el suelo, producto de variaciones en el estado de oxidación, variaciones en el $\mathrm{pH}$, inhibición de los mecanismos de absorción en virtud de una carga energética baja de las membranas celulares y a la propia reducción de la superficie de absorción de las raíces (Drew, 1997; de Carvalho e Ishida, 2002).

Los tratamientos con los mejores resultados correspondieron a la presencia de la bacteria Azospirillum brasilense y se resalta además el efecto negativo en aquellos tratamientos donde la presencia del oxígeno era un factor limitante, incluso donde el microorganismo se encontraba presente, lo que hace indicar que en aquellos suelos con limitaciones de niveles de oxígeno, son limitantes también para el normal desarrollo de Azospirillum brasilense, sobre el asunto algunos autores han encontrado que este microorganismo puede enriquecer el contenido de nitrógeno de la planta por fijación cuando los niveles de nitrógeno son bajos y los de oxígeno son óptimos en el suelo (Bothe et al., 1981), aún cuando para esta cepa microbiana se resalta un alto grado de especificidad (Pérez y Casas, 2005).

El mayor desarrollo en profundidad del sistema radical en presencia de la bacteria Azospirillum brasilense, se debe al efecto rizosférico de mejora del desarrollo radical de la planta que ejerce el microorganismo, disminuyendo los costos de producción por el ahorro de fertilizantes minerales (Saad et al., 1996), debido en parte al incremento de los pelos radicales que provoca esta bacteria, la inducción de auxinas, giberelinas y citoquininas, que estimulan hormonalmente el crecimiento de la raíz (Vlassak y Reyders, 1977; Vose, 1983).

El empleo agronómico de la bacteria Azospirillum sp., constituye una práctica sustentable que permite mejorar los parámetros de calidad de los plantines en hortalizas, aumentando la precocidad de los mismos (Hadas y Okon, 1987; Correa et al., 2002). Este microorganismo es una rizobacteria promotora del crecimiento vegetal asociada con plantas que no nodulan, presentando gran potencial como biopreparado, permitiendo reducir entre 40 y $50 \%$ los fertilizantes químicos, al ser descrito en numerosas investigaciones que la inoculación con esta bacteria en gramíneas de interés alimenticio provoca un incremento en sus rendimientos agrícolas (Tien et al., 1979; Pérez y Casas, 2005).

Con la suma de las dos componentes en la representación biplot del análisis de componentes principales (Figura 2), se explica el $100 \%$ de variabilidad total, lo que demuestra el alto grado de correlación existente entre las cuatro variables estudiadas. Los tratamientos donde el suelo no se

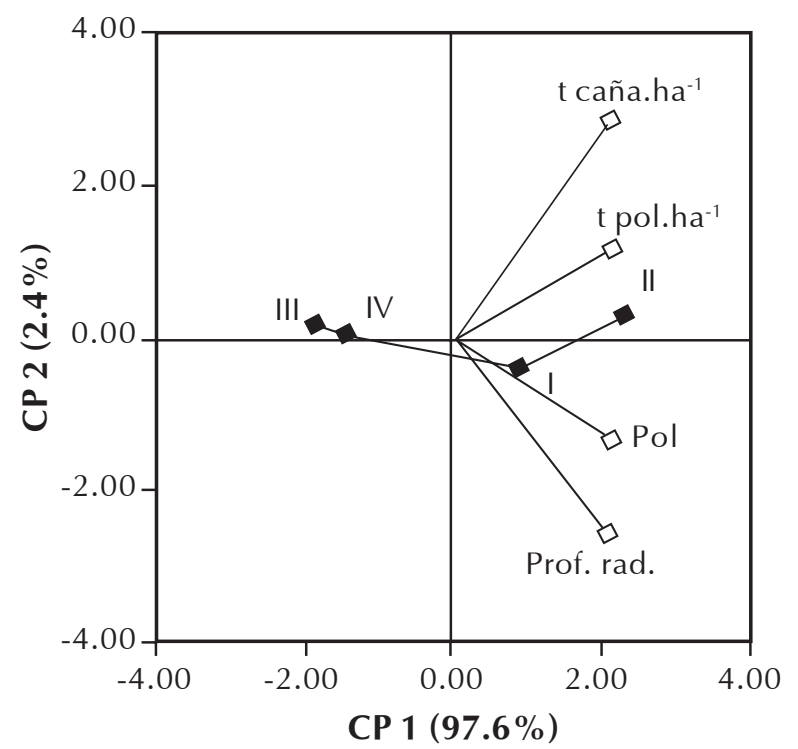

Figura 2. Representación biplot del análisis de componentes principales con la inclusión de los árboles de recorrido mínimo. 
sobresaturó de agua, es decir el I y II, se caracterizaron por presentar los valores mayores en las variables estudiadas, contrario a lo alcanzado por los tratamientos III y IV, con los valores menores, corroborado por el árbol de recorrido mínimo en que la menor distancia o mayor similitud en base a las cuatro variables estuvo representada en los tratamientos III y IV por un lado y entre el I y II por el otro, lo que demuestra que la carencia de oxígeno en el suelo por sobresaturación de agua es un elemento que determina la respuesta agroproductiva de la caña de azúcar y la escasa influencia benéfica de la bacteria Azospirillum brasilense cepa 8 del INICA en estas condiciones.

\section{CONCLUSIONES}

- La presencia de la bacteria Azospirillum bresilense, cepa 8 del INICA en condiciones de suelo no sobresaturado de agua, contribuye al incremento de indicadores del crecimiento y desarrollo de la caña de azúcar, variedad C120-78.

- La sobresaturación de agua en el suelo es un elemento inhibitorio del crecimiento y desarrollo de la caña de azúcar, variedad C120-78, aún en presencia de la bacteria Azospirillum bresilense, cepa 8 del INICA.

\section{BIBLIOGRAFÍA}

Bothe, N.; Tennigkeit, J. y Eisbrenmer, G. 1981. Transformation of inorganic nitrogen by Azospirillum spp. Archives of Microbiology 130: 96-100

Correa, O.; Moccia, S. y Romero, A. 2002. Producción de plantines de tomate: Condiciones de aplicación de azospirillum sp. Proyecto UBACYT01/G009. Cátedra de Microbiología, Facultad de Agronomía. Universidad de Buenos Aires, Argentina

De Carvalho, C. e Ishida, F. 2002. Responses of young pijuayo plants (Bactris gasipaes Kunth) to flooding. Pesquisa Agropecuária Brasileira 37(9):12311237

Deren, C.; Cherry, R. y Zinder, G. 1993. Effect of flooding on selected sugarcane clones. Journal American Society of Sugar Cane Technology 13:22-26

Drew, M. 1997. Oxygen deficiency and metabolism: injury and acclimation under hypoxia and anoxia. Annual Review of Plant Physiology and Plant Molecular Biology 48:223-250

Driessen, P.; Deckers, J.; Sparargaren, O. y Nachtergaele, F. 2001. Lecture notes on the major soils of the world. World Soil Resources Reports, 94, FAO, Roma, 334 pp.

Eckert, B.; Weber, O.; Kirchhof, G.; Halbritter, A.; Stofields, M. y Hartman, A. 2001. Azospirillum doebereineare sp. nov., a nitrogen - fixing bacterium associated with the $\mathrm{C}(4)$ - grass Miscanthus. International Journal of Systematic and Evolutionary Microbiology 51:17-26

Fida, M.; Alam. H.; Jabber, M.; Begur, M y Miah, M. 2003. Effects of waterlogging on juice quality and yield of sugarcane. Pakistan Journal of Biological Sciences 6(3):11511155 
Gabriel, K. 1971. The biplot graphic display of matrices with applications to principal components analysis. Biometrika 58(3):453-467

Gower, J. y Ross, P. 1969. Minimun spanning tress and single cluster analysis. Applied Statistics 18:54-64

Hadas, R. y Okon, V. 1987. Effect of Azospirillum brasilense inoculation on root morphology and respitation in tomato seedlings. Biology and Fertility of Soils 5(3):241-247

Hotelling, H. 1933. Analysis of a complex of statistical variables into principal components. Journal of Educational Psychology 24:498-520

Humbert, R. 1965. El cultivo de la caña de azúcar. Editorial Universitaria, La Habana, 785 pp.

INICA. 2002. Normas y procedimientos del programa de mejoramiento genético de la caña de azúcar en Cuba. Boletín No. 1 Cuba \& Caña-INICA, Instituto Nacional de Investigaciones de la Caña de Azúcar, La Habana, 315 pp.

Inman-Bamber, N.; Bonnett, G.; Smith, D. y Thorburn, P. 2004: Sugarcane physiology: Integrating from cell to crop to advance sugarcane production. Field Crops Research 92(2-3):115-117

Kolensnikov, V. 1971. The root system of fruit plant. MIR, Moscow, 269 pp.

Krautman, S. 1959. Observacoes do sistema radicular da Cana IANE-C46117 en solo de Baixada (Varzea). Boletín Técnico do Instituto Agronómico do Norte 6:25-31
Martín, J.; Gálvez, G.; De Armas, R.; Espinosa, R.; Vigoa, R. y León, A. 1987. La Caña de Azúcar en Cuba. Editorial Científico Técnica, La Habana, 612pp.

Morrison, D. 1979. Multivariate statistics. D. F. Morrison, John Wiley and Sons, New York, 414 pp.

Pérez, J. y Casas, M. 2005. Estudio de la interacción planta - Azospirilum en el cultivo caña de azúcar (Saccharum spp. híb.). Cultivos Tropicales 26(4):13-19

Saad, M.; Ali-Sabuddin, A.; Yunus, A. y Shamsuddin, Z. 1996. Performance of sweetpotato variety OP MSS 5 inoculated with Azospirillum on sandy ten tilling soil. Sweetpotato 2:107-119

SAS Institute. 2000. Statistical Analysis System. SAS Institute. User's guide. Version 8.0. SAS Institute, Inc., Cary, North Carolina, 3884 pp.

Soil Survey Staff. 2003. Claves para la taxonomía de suelos. Colegio de Postgraduados, Chapingo, 306 pp.

Vlassak, K y Reyaders, L. 1977. Associative dinitrogen fixiation in temperature regions. In isotipcs in biological dinitrogen fixiation. Proceedings of the Advisory Group, International Atomic Energy, Viena, p 71

Vose, B. 1983. Developments in nonlegume N2-fixing systems. Canadian Journal of Microbiology 29:837-849 